\title{
Determination of the Long Period Nutation Terms from Optical Astrometry and VLBI Data
}

\author{
P. Yaya, C. Bizouard \\ Observatoire de Paris, 61 avenue de l'Observatoire, 75014 Paris, France \\ C. Ron \\ Astronomical Institute, Academy of Sciences of the Czech Republic, \\ Bočni II, 14131 Prague 4, Czech Republic
}

\begin{abstract}
A 100-year long optical astrometric series of the Earth Orientation Parameters produced by a Czech team (Vondrák et al., 1998) has been analysed in order to determine components of nutation. Our interest is mostly focused on the long periodic terms: 18.6-year term, 9.3-year term and linear trend, still correlated in VLBI series which cover only the last 20 years. A comparison has been made with the corresponding values determined from the VLBI series.
\end{abstract}

\section{Introduction}

The VLBI technique allows us to obtain high precision estimation of some nutation terms (periods shorter than 9 years) as we reach now a sub-milliarcsecond precision. But the longest ones, especially the principal term (18.6 years), still have a non-negligeable correlation with precession. Their amplitudes cannot be estimated with great reliability.

Taking into account theoretical constraints could partially solve the problem (Souchay et al., 1995). Another way is to use a longer time-span of data. The latter possibility has been applied in the present work. Actually, we use two series of celestial pole offsets from optical atrometry $(\mathrm{OA})$, covering nearly one century.

In order to compare VLBI and OA data (and also both OA series), a spectral analysis on various subsets has been undertaken, as well as several estimates of the main nutation terms using a least-squares method.

\section{The observations}

We have used VLBI celestial pole offsets series of the US Naval Observatory which ranges from 1979 to 1999 . As the data of the first five years are neither numerous nor precise enough, we chose to analyse only the post-1984 data.

For the optical series two versions (OA97 and OA99) covering the 1899.71992.0 time span are available. The OA99 series includes more observations than 
OA97 (4.5 million instead of 4.3 million) and is referred to a slightly different reference frame due to proper motions corrections.

In Figure 1 are displayed both VLBI and OA series, restricted to the nutation in longitude. A similar pattern has been obtained in obliquity. Note the weaker dispersion after 1956 for the OA series, due to the adoption of atomic time.

The precision of the observations are of course very different: 30 mas for $\mathrm{OA}$ before 1956, 10 mas after 1956, whereas the VLBI uncertainties are smaller than 0.5 mas. But, as it has been already said, each data set has its own advantage: precision for VLBI, long time-span for OA.

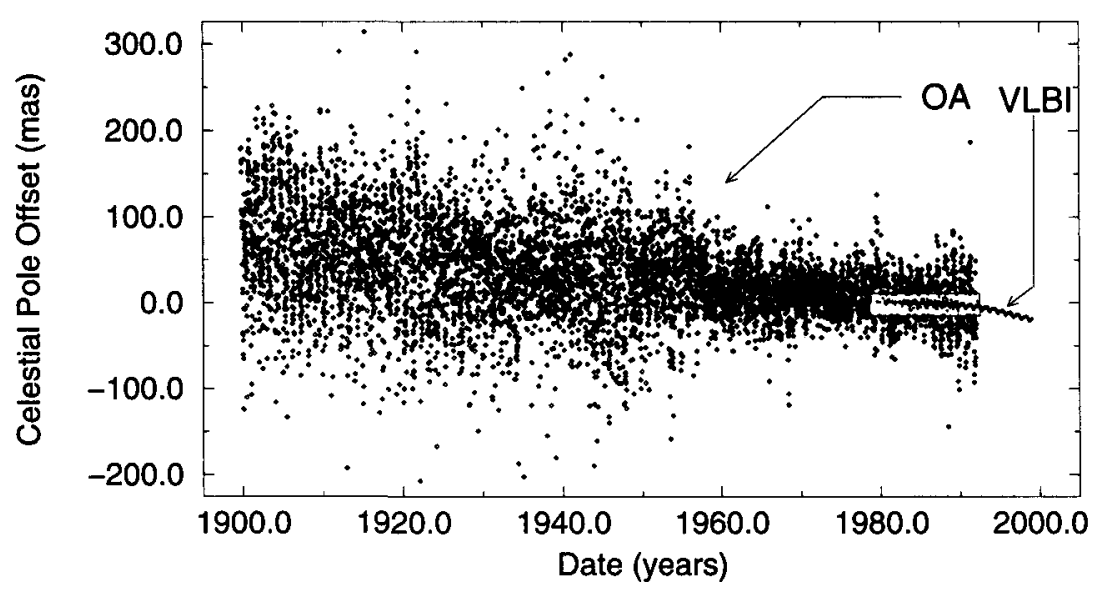

Figure 1. VLBI and Optical Astrometry (OA) series: corrections to the nutation in longitude $\Delta \Psi \sin \varepsilon_{0}$ (some OA points have been deleted near the VLBI data).

\section{Spectral analysis}

\subsection{Spectral analysis of the VLBI data}

We performed a complex FFT analysis in order to identify prograde and retrograde periodic terms in the data. Figure 2 shows the VLBI spectrum obtained with this technique. The ordinate axis is the $\log _{10}$ of the squared amplitude.

The long periods ( 1 year and more) stand out clearly at the center of the spectrum. We can distinguish a strong annual retrograde signal, a semiannual prograde one, as well as a long periodic peak. But we cannot affirm whether the latter comes from the 9.3 or the 18.6 years term. This lack of resolution is due to the insufficient time-span covered by VLBI.

One can see shorter periods also. The 13.6-day and the 27.5-day are present. But we cannot still distinguish between the 13.63 and the 13.66 days since the resolution is not sufficient. 


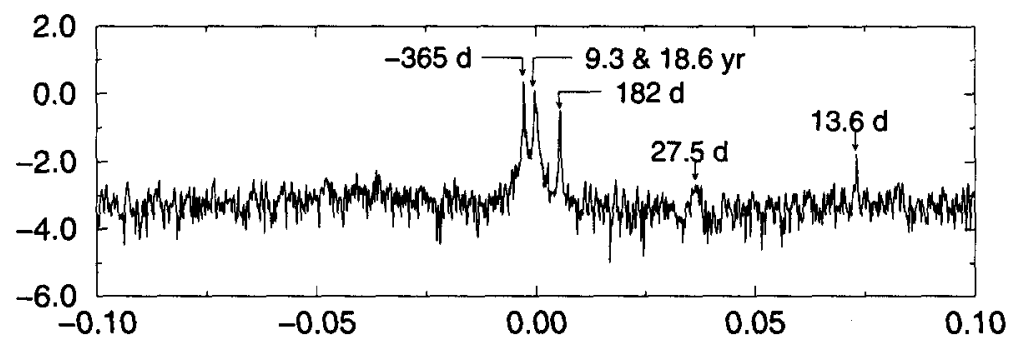

Figure 2. VLBI spectrum: $\log _{10}\left(\right.$ amplitude $\left.{ }^{2}\right)$ versus frequency in cycles/day.

\subsection{Spectral analysis of the optical astrometry data}

In Figure 3 we present different OA spectra. The upper ones stand for the 1997 series and the lower ones for the 1999 series. For each series we have analysed the whole time-span (figures on the left) as well as the post-1956 data (figures on the right).

In all of them, the annual correction is the strongest. It is interesting to note that, contrary to the VLBI case, this annual term is mostly prograde. This term could be due to a mismodeling of the refraction, which varies annually at a given location.

The 1899-1992 series exhibit a lot of harmonic terms (18.6-years, semiannual, ter-annual...) but their amplitudes become smaller in the 1956-1992 ones. No significant short period term (such as 13.6-days) has been detected.

\section{Estimates of the long period terms}

We have then estimated the coefficients of the nine principal terms (the same as in Vondrák et al. (1995 \& 1998)), by adjusting them with a least-squares method. The nine periods are: 6798.35 days (18.6 years), 3399.18 days $(9.3$ years), 365.25 days, 182.62 days, 177.84 days, 121.75 days, 27.55 days, 13.66 days and 13.63 days

We used the following model of nutation:

$$
\begin{aligned}
\Delta \Psi & =\sum_{\alpha} \Delta \Psi_{s}^{\alpha} \sin \theta(t)+\Delta \Psi_{c}^{\alpha} \cos \theta(t)+a_{\Psi}\left(t-t_{0}\right)+b_{\Psi}, \\
\Delta \varepsilon & =\sum_{\alpha} \Delta \varepsilon_{s}^{\alpha} \sin \theta(t)+\Delta \varepsilon_{c}^{\alpha} \cos \theta(t)+a_{\varepsilon}\left(t-t_{0}\right)+b_{\varepsilon}
\end{aligned}
$$

where $\theta(t)$ is the astronomical argument of the nutation $\alpha$ and $t_{0}$ stands for the date $\mathrm{J} 2000.0$.

If we first compare the two optical series (see Table 1), we note that the estimates are quite different, especially the out-of-phase terms $\Delta \Psi_{c}$ for 9.3 years and the constant one in longitude.

The comparison with VLBI shows a significant agreement for all the 18.6years terms, except for $\Delta \Psi_{c}$ which presents an unexplained opposite sign. Nev- 

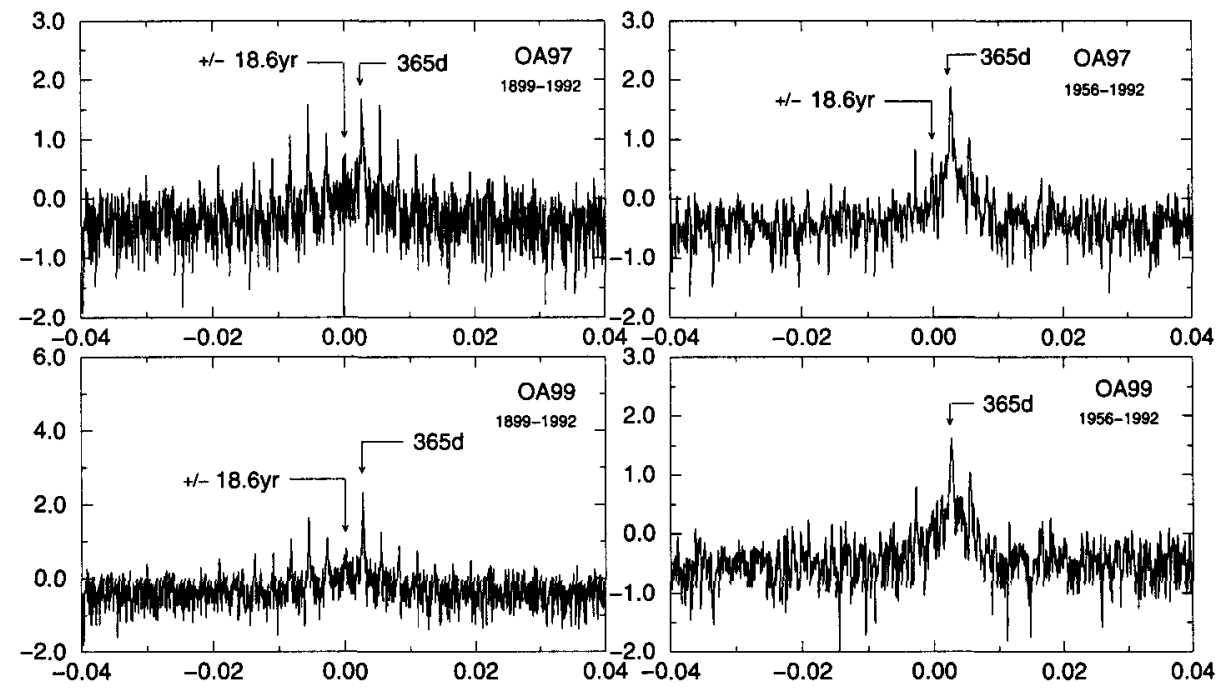

Figure 3. Optical astrometry spectra: $\log _{10}\left(a m p l i t u d e^{2}\right)$ versus frequency in cycle/day.
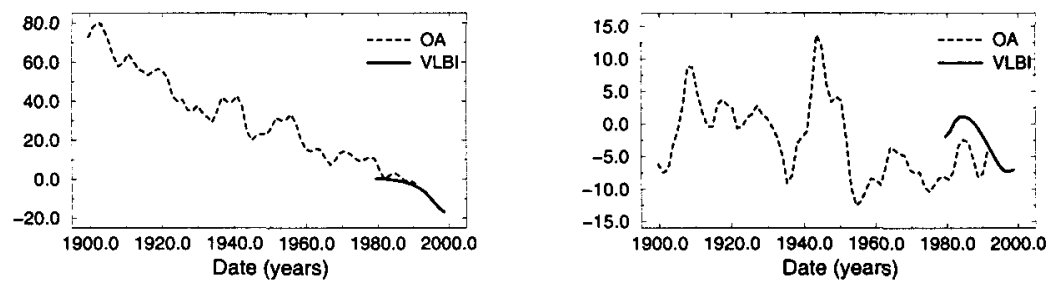

Figure 4. Corrections in mas to celestial pole offsets in longitude (left) and obliquity (right) after a 500 days filtering.

ertheless, an interesting result is the equivalent uncertainty ( $\sim 0.04$ mas in longitude and $\sim 0.02$ mas in obliquity), for the secular term from VLBI and OA.

For the OA97 series, the agreement is generally better (see the remarkably small difference in $\Delta \Psi_{s}$ or in $\Delta \varepsilon_{c}$ for the 18.6-years term), and the uncertainties are smaller.

\section{Filtering}

In order to understand some of the disagreements between VLBI and OA estimates, it would be interesting to visualize the long term evolution. In Figure 4, we show the long-period variations for both series. This has been made possible by applying a high-pass Gaussian filter to the OA and VLBI series (the cut-off period is 500 days). 
Table 1. Corrections to the amplitudes of some nutation terms (in mas)

\begin{tabular}{|c|c|c|c|c|c|}
\hline & & $\Delta \Psi_{s}$ & $\Delta \Psi_{c}$ & $\Delta \varepsilon_{s}$ & $\Delta \varepsilon_{c}$ \\
\hline \multirow{3}{*}{$18.6 \mathrm{yrs}$} & OA 97 & $-6.77 \pm 1.31$ & $-3.58 \pm 1.34$ & $3.34 \pm 0.50$ & $3.18 \pm 0.51$ \\
\hline & OA 99 & $-4.31 \pm 1.33$ & $-2.16 \pm 1.36$ & $2.76 \pm 0.51$ & $1.83 \pm 0.52$ \\
\hline & VLBI & $-6.79 \pm 0.06$ & $3.35 \pm 0.20$ & $1.48 \pm 0.02$ & $3.02 \pm 0.08$ \\
\hline \multirow{3}{*}{$9.3 \mathrm{yrs}$} & OA 97 & $3.54 \pm 1.27$ & $0.89 \pm 1.28$ & $1.31 \pm 0.50$ & $-1.33 \pm 0.50$ \\
\hline & OA 99 & $3.12 \pm 1.29$ & $2.41 \pm 1.30$ & $1.22 \pm 0.51$ & $-1.10 \pm 0.51$ \\
\hline & VLBI & $1.06 \pm 0.07$ & $-0.20 \pm 0.04$ & $0.13 \pm 0.03$ & $-0.32 \pm 0.02$ \\
\hline \multirow{4}{*}{$\begin{array}{l}\text { secular } \\
\text { trend } a \\
(\mathrm{mas} / \mathrm{yr})\end{array}$} & & \multicolumn{2}{|c|}{$\Delta \bar{\Psi}$} & \multicolumn{2}{|c|}{$\Delta \varepsilon$} \\
\hline & OA 97 & \multicolumn{2}{|c|}{$-1.54 \pm 0.04$} & \multicolumn{2}{|c|}{$-0.13 \pm 0.02$} \\
\hline & OA 99 & \multirow{2}{*}{\multicolumn{2}{|c|}{$\begin{array}{l}-2.16 \pm 0.05 \\
-3.00 \pm 0.04\end{array}$}} & \multicolumn{2}{|c|}{$-0.09 \pm 0.02$} \\
\hline & VLBI & & & \multicolumn{2}{|c|}{$-0.19 \pm 0.02$} \\
\hline \multirow{3}{*}{ const. $b$} & OA 97 & \multicolumn{2}{|c|}{$-15.12 \pm 1.62$} & \multicolumn{2}{|c|}{$-9.49 \pm 0.64$} \\
\hline & OA 99 & \multicolumn{2}{|c|}{$-31.03 \pm 1.67$} & \multirow{2}{*}{\multicolumn{2}{|c|}{$\begin{array}{l}-9.17 \pm 0.66 \\
-4.69 \pm 0.12\end{array}$}} \\
\hline & VLBI & \multicolumn{2}{|c|}{$-42.96 \pm 0.29$} & & \\
\hline
\end{tabular}

Visually, the 18.6-year oscillation does not stand out and we can see inexplicable variations with a smaller period (6 or 7 years) which do not correspond to any known nutation. The positive point is that the VLBI and OA series seems to have a similar evolution, even though there is an edge effect at the end of OA data in obliquity.

\section{Conclusion}

Optical astrometry series of celestial pole offsets reveal unexplainable variations of 6-7 year period. It's a challenge to determine whether they result from measurement errors or are real effects.

Even if those variations prevent us from having exact estimations of the nutation coefficients now, this study proves that with further work, the optical series can give us very useful information concerning the secular trend or the constant term of nutation.

Maybe an eventual combination of OA and VLBI series, like the one begun in Bizouard et al. (1997), could be a good solution to our lack of information about long periodic variations in the nutation phenomena.

\section{References}

Bizouard, C., Capitaine, N., Ron, C., Vondrák, J. 1997, Dynamics and Astrometry of Natural and Artificial Celestial Bodies, 481.

Souchay, J., Feissel, M., Bizouard, C., Capitaine, N., Bougeard, M. 1995, $A \mathscr{E} A$, $299,277$.

Vondrák, J., Ron, C., Pešek, I., Čepek, A. 1995, A\&A, 297, 899 
Vondrák, J., Pešek, I., Ron, C., Cepek, A. 1998, Publication of the Astronomical Institute of the Academy of Sciences of the Czech Republic, No. 87. 